\title{
Spacetime singularity resolution in Snyder noncommutative space
}

\author{
M. A. Gorji* and K. Nozari ${ }^{*}$ \\ Department of Physics, Faculty of Basic Sciences, \\ University of Mazandaran, \\ P. O. Box 47416-95447, Babolsar, Iran \\ B. Vakil用 \\ Department of Physics, Chalous Branch, \\ Islamic Azad University, \\ P.O. Box 46615-397, Chalous, Iran
}

\begin{abstract}
Inspired by quantum gravity proposal, we construct a deformed phase space which supports the UV and IR cutoffs. We show that the Liouville theorem is satisfied in the deformed phase space which allows us to formulate the thermodynamics of the early Universe in the semiclassical regime. Applying the proposed method to the Snyder noncommutative space, we find a temperature dependent equation of state which opens a new window for natural realization of inflation as a phase transition from quantum gravity regime to the standard radiation dominated era. Also we obtain finite energy and entropy densities for the Universe, when at least the Weak Energy Condition is satisfied. We show that there is a minimum size for the Universe which is proportional to the Planck length and consequently the Big Bang singularity is removed.
\end{abstract}

PACS numbers: 04.60.Bc, 98.80.Cq

Key Words: Quantum Gravity Phenomenology, Phase Transition, Big Bang Singularity

\section{INTRODUCTION}

The dynamics of the Universe in the Standard Big Bang scenario is governed by the Einstein's general theory of relativity. If general relativity is used to describe the observed Universe, the model requires huge fine-tuned initial conditions [1]. Such an initial conditions can be accommodated by the standard model, but of course, they can not be explained in this framework. Inflationary scenario, an accelerating phase before the nucleosynthesis era, can resolve this problem in a novel way [2]. Such a scenario can be realized, for instance, from a GUTs symmetry breaking phase transition $S U(5) \rightarrow S U(3) \times S U(2) \times U(1)$ around $10^{15} \mathrm{GeV}$ [3]. While inflation solves the initial value problem, it is not a natural prediction of general relativity. More precisely, general relativity is a classical theory and is applicable at sub-Planckian curvatures. On the other hand, inflation occurs in the quantum gravity regime and so it is plausible to expect that the problem of initial condition will be naturally addressed in the framework of full quantum gravity theory. In the absence of a full quantum theory of gravity, we don't know the exact dynamical equations governing on the early Universe. Nevertheless, there are some candidates such as string theory and loop quantum gravity which revealed some unknown aspects of the quantum gravity. For instance, existence of a minimal length and a minimal momentum which induce ul-

\footnotetext{
* m.gorji@stu.umz.ac.ir

† knozari@umz.ac.ir

$\ddagger$ b-vakili@iauc.ac.ir
}

traviolet (UV) and infrared (IR) cutoffs respectively, are common addresses of alternative candidates for quantum gravity proposal [4, 5, 6, 7, 8, 9].

Einstein equations for the Universe, including the Friedmann and Raychaudhuri equations, can not be solved without supplementing an equation of state. The equation of state is determined by statistical analysis of the particles in the Universe. The question then arises: is the equation of state in quantum gravity regime the same as the low energy regime ones? We focus on this question in the present study by considering a deformed phase space that includes some phenomenological aspects of the quantum gravity proposal in semiclassical regime. On the other hand, in the standard model of cosmology the adiabatic expansion condition implicitly leads to the Big Bang singularity when one reverses the expansion history. We show here that quantum gravity effects modify the adiabatic expansion condition leading trivially to a nonsingular early Universe. Interestingly this resolves the large entropy density problem too.

The structure of the paper is as follows: In Section 2, we introduce a kinematical phase space $\tilde{\Gamma}$ which is consistent with quantum gravity proposal and supports the existence of the UV and IR cutoffs. In the dynamical level, we show that the Liouville theorem is satisfied in the deformed phase space, which ensures that the number of quantum states is invariant under the time evolution of the system. In Section 3, we formulate the thermostatistics in noncommutative phase space and we study some consequences of the model in the early Universe cosmology. In Section 4, we apply the proposed model to the Snyder noncommutative space. Section 5 is devoted to the conclusions. 


\section{THE DEFORMED PHASE SPACE}

The spacetime manifold structure is significantly affected by quantum gravity effects in the high energy regime. All alternative candidates of quantum gravity suggest some deformations of the algebraic structure in such a way that the system under consideration to be UV and IR regularized. These deformations can be addressed properly through the modified Heisenberg commutation relations. The Snyder noncommutative spaces are the well-known example which can be realized from the modified commutation relations and also are naturally UV/IR-regularized [9, 10]. The generalized uncertainty principle is another proposal which is suggested in the context of the string theory and supports the existence of minimal length (UV cutoff) and minimal momentum (IR cutoff) [4, 5]. Also, polymer quantization is an effective approach to the loop quantum gravity [11] which suggests the direct deformation to the phase space variables through a process known as polymerization [12, 13, 14]. In what follows we formulate the kinematics and dynamics of representative points in a phase space with natural cutoffs.

\section{A. Kinematics}

Heisenberg algebra will be deformed in quantum gravity regime. The deformed Heisenberg algebra leads to the deformed Poisson algebra in the classical limit through a standard relation $\frac{1}{i}[\hat{A}, \hat{B}] \rightarrow\{A, B\}$ [15]. The most general form of the noncanonical symplectic structure on phase space $\tilde{\Gamma}$ of dimension $2 D$ is

$$
\begin{aligned}
& \left\{q_{i}, q_{j}\right\}=f_{i j}(q, p), \\
& \left\{q_{i}, p_{j}\right\}=g_{i j}(q, p), \\
& \left\{p_{i}, p_{j}\right\}=h_{i j}(q, p),
\end{aligned}
$$

where $i, j=1,2, \ldots, D$. Here, $q$ and $p$ are the positions and conjugate momenta respectively and $f_{i j}, g_{i j}$ and $h_{i j}$ are the differentiable functions which determine the deformed Poisson algebra on $\tilde{\Gamma}$. Specifying particular forms for these functions, one recovers the well-known noncommutative algebras such as the Snyder and the Moyal algebras (see appendix A).

The above deformed Poisson brackets should have the same properties as the usual Poisson brackets, that is, they should be antisymmetric, bilinear, and satisfy the Leibnitz rule and the Jacobi identity. Clearly, $f_{i j}$ and $h_{i j}$ should be totaly antisymmetric $f_{i j}=-f_{j i}$ and $h_{i j}=-h_{j i}$ through the antisymmetric property of the Poisson brackets. For two arbitrary functions $U\left(\zeta_{a}\right)$ and $V\left(\zeta_{a}\right)$ where $\zeta_{a}=\left(q_{i}, p_{i}\right)$ with $a=1,2, \ldots, 2 D$, the Poisson bracket reads

$$
\{U, V\}_{\zeta}=\left\{\zeta_{a}, \zeta_{b}\right\} \frac{\partial U}{\partial \zeta_{a}} \frac{\partial V}{\partial \zeta_{b}} .
$$

Expanding the above relation in terms of the phase space variables $q$ and $p$ and using relations (1) one obtains

$$
\begin{aligned}
\{U, V\}_{[q, p]}= & g_{i j}\left(\frac{\partial U}{\partial q_{i}} \frac{\partial V}{\partial p_{j}}-\frac{\partial U}{\partial p_{j}} \frac{\partial V}{\partial q_{i}}\right) \\
& +f_{i j} \frac{\partial U}{\partial q_{i}} \frac{\partial V}{\partial q_{j}}+h_{i j} \frac{\partial U}{\partial p_{i}} \frac{\partial V}{\partial p_{j}}
\end{aligned}
$$

Also, the Jacobi identity

$$
\{U,\{V, W\}\}+\{V,\{W, U\}\}+\{W,\{U, V\}\}=0
$$

is satisfied for any functions $U(q, p), V(q, p)$, and $W(q, p)$ with continuous second derivative. Substituting phase space variables $q$ and $p$ into the relation (4) gives four independent equations

$$
\begin{aligned}
& \left\{q_{i},\left\{q_{j}, q_{k}\right\}\right\}+\left\{q_{j},\left\{q_{k}, q_{i}\right\}\right\}+\left\{q_{k},\left\{q_{i}, q_{j}\right\}\right\}=0, \\
& \left\{q_{i},\left\{q_{j}, p_{k}\right\}\right\}+\left\{q_{j},\left\{p_{k}, q_{i}\right\}\right\}+\left\{p_{k},\left\{q_{i}, q_{j}\right\}\right\}=0 \\
& \left\{q_{i},\left\{p_{j}, p_{k}\right\}\right\}+\left\{p_{j},\left\{p_{k}, q_{i}\right\}\right\}+\left\{p_{k},\left\{q_{i}, p_{j}\right\}\right\}=0 \\
& \left\{p_{i},\left\{p_{j}, p_{k}\right\}\right\}+\left\{p_{j},\left\{p_{k}, p_{i}\right\}\right\}+\left\{p_{k},\left\{p_{i}, p_{j}\right\}\right\}=0 .
\end{aligned}
$$

Substituting from (1) and using (3), the relations (5) give the constraints on the functions $f_{i j}, g_{i j}$, and $h_{i j}$

$$
\begin{array}{r}
f_{i m} \frac{\partial f_{j k}}{\partial q_{m}}+g_{i m} \frac{\partial f_{j k}}{\partial p_{m}}+f_{j m} \frac{\partial f_{k i}}{\partial q_{m}}+g_{j m} \frac{\partial f_{k i}}{\partial p_{m}} \\
+f_{k m} \frac{\partial f_{i j}}{\partial q_{m}}+g_{k m} \frac{\partial f_{i j}}{\partial p_{m}}=0, \\
f_{i m} \frac{\partial g_{j k}}{\partial q_{m}}+g_{i m} \frac{\partial g_{j k}}{\partial p_{m}}-f_{j m} \frac{\partial g_{i k}}{\partial q_{m}}-g_{j m} \frac{\partial g_{i k}}{\partial p_{m}} \\
-g_{m k} \frac{\partial f_{i j}}{\partial q_{m}}+h_{k m} \frac{\partial f_{i j}}{\partial p_{m}}=0, \\
f_{i m} \frac{\partial h_{j k}}{\partial q_{m}}+g_{i m} \frac{\partial h_{j k}}{\partial p_{m}}+g_{m j} \frac{\partial g_{i k}}{\partial q_{m}}-h_{j m} \frac{\partial g_{i k}}{\partial p_{m}} \\
-g_{m k} \frac{\partial g_{i j}}{\partial q_{m}}+h_{k m} \frac{\partial g_{i j}}{\partial p_{m}}=0 \\
-g_{m i} \frac{\partial h_{j k}}{\partial q_{m}}+h_{i m} \frac{\partial h_{j k}}{\partial p_{m}}-g_{m j} \frac{\partial h_{k i}}{\partial q_{m}}+h_{j m} \frac{\partial h_{k i}}{\partial p_{m}} \\
-g_{m k} \frac{\partial h_{i j}}{\partial q_{m}}+h_{k m} \frac{\partial h_{i j}}{\partial p_{m}}=0 .
\end{array}
$$

We will see that the measure of the phase space $\tilde{\Gamma}$ is different from the measure of the usual phase space $\Gamma$. To show this fact, consider a general noncanonical transformation of the phase space

$$
(Q, P) \rightarrow(q, p)
$$

where the variables $Q$ and $P$ obey the nondeformed Poisson algebra on the phase space $\Gamma$

$$
\left\{Q_{i}, Q_{j}\right\}=0, \quad\left\{Q_{i}, P_{j}\right\}=\delta_{i j}, \quad\left\{P_{i}, P_{j}\right\}=0,
$$

while the variables $q=q(Q, P)$ and $p=p(Q, P)$ belong to the deformed phase space $\tilde{\Gamma}$ and satisfy the deformed Poisson algebra (11). The Jacobian of transformation (7) 
in $2 D$-dimensional classical phase space can be expanded in terms of the Poisson brackets as [16, 17],

$$
\begin{aligned}
& J(q, p)=\frac{\partial(q, p)}{\partial(Q, P)}= \\
& \frac{1}{2^{D} D !} \sum_{i_{1} \ldots i_{2 D}=1}^{2 D} \epsilon_{i_{1} . . i_{2 D}}\left\{X_{i_{1}}, X_{i_{2}}\right\} \ldots\left\{X_{i_{2 D-1}}, X_{i_{2 D}}\right\}
\end{aligned}
$$

where $\epsilon$ denotes the Levi-Civita symbol and $X_{i}$ denotes the phase space variables so that for odd $i$ it is a coordinate $q_{i}$ and for even $i$ it is a conjugate momenta $p_{i}$. The Jacobian $J(q, p)$ induces the UV and IR cutoffs in the high and low energy regimes respectively. The deformation to the measure of the phase space $\tilde{\Gamma}$ can be obtained by means of the Jacobian (9) as

$$
\int_{\Gamma}(\ldots) d \omega(Q, P) \longrightarrow \int_{\tilde{\Gamma}}(\ldots) \frac{d \tilde{\omega}(q, p)}{J(q, p)}
$$

where $d \omega(Q, P)$ is the infinitesimal volume of the $2 D$ dimensional usual phase space $\Gamma$ and $d \tilde{\omega}(q, p) / J(q, p)$ is its counterpart in the deformed phase space $\tilde{\Gamma}$. It is important to note that the deformed phase space volume $d \tilde{\omega}(q, p) / J(q, p)$ should be invariant under the time evolution of the system. We consider this issue in the next section and we show that the Liouville theorem is satisfied in the deformed phase space $\tilde{\Gamma}$. In general, the phase spaces $\Gamma$ and $\tilde{\Gamma}$ topologically may represent different symplectic manifolds, but the problem arises is that how these two manifolds coincide in the limit of the low energy regime? This problem arises, for example, in polymer framework which can be resolved by detailed analysis of the continuous limit of the corresponding theory 12, 13]. In the present study these manifolds are topologically the same, though at the boundaries they may behave differently because of, for instance, existence of minimal length, minimal momentum and maximal momentum which may affect the range of integrals in relation (10).

Although the ultimate form of the Jacobian $J(q, p)$ will be specified just after formulating the full quantum gravity theory, but effective theories to quantum gravity proposal have proposed some candidates for this quantity 18, 19, 20, 21, 22, 23, 24]. Moreover, the deformation such as the relation (10), can be deduced without demanding modified commutation relations. For instance, the coherent states approach to the spacetime noncommutativity provides a direct deformation to the phase space which is equivalent to $J(q, p)^{-1}=e^{-\sigma q^{2}-\theta p^{2}}[7,[8]$ (see also Appendix A), where $\sigma$ and $\theta$ are the noncommutative deformation parameters that induce the IR and UV cutoffs respectively.

\section{B. Dynamics and the Liouville Theorem}

The next issue now is to consider the dynamics of the model. The deformed measure

$$
\frac{d \tilde{\omega}(q, p)}{J(q, p)},
$$

determines the number of quantum states in the phase space $\tilde{\Gamma}$. So, the deformed volume $d \tilde{\omega}(q, p) / J(q, p)$ should be invariant under the time evolution of the system to ensure that the Liouville theorem is satisfied and consequently the number of microstates remains unchanged.

Time evolution of any function of the phase space $U(q, p)$ in Hamiltonian formalism can be represented by the Poisson brackets

$$
\frac{d U}{d t}=\{U, \mathcal{H}\}
$$

where $\mathcal{H}(q, p)$ is the Hamiltonian of the system. The equations of motion can be obtained from the relations (3) and (12)

$$
\begin{gathered}
\dot{q}_{i}=\left\{q_{i}, \mathcal{H}\right\}=f_{i j} \frac{\partial \mathcal{H}}{\partial q_{j}}+g_{i j} \frac{\partial \mathcal{H}}{\partial p_{j}}, \\
\dot{p}_{i}=\left\{p_{i}, \mathcal{H}\right\}=-g_{j i} \frac{\partial \mathcal{H}}{\partial q_{j}}+h_{i j} \frac{\partial \mathcal{H}}{\partial p_{j}},
\end{gathered}
$$

Consider an infinitesimal transformation of the phase space variables $q_{i}$ and $p_{i}$

$$
\begin{aligned}
& q_{i}^{\prime}=q_{i}+\delta q_{i}, \\
& p_{i}^{\prime}=p_{i}+\delta p_{i},
\end{aligned}
$$

where $\delta q_{i}$ and $\delta p_{i}$ evolve through relations (13) as

$$
\begin{gathered}
\delta q_{i}=\left(f_{i j} \frac{\partial \mathcal{H}}{\partial q_{j}}+g_{i j} \frac{\partial \mathcal{H}}{\partial p_{j}}\right) \delta t, \\
\delta p_{i}=-\left(g_{j i} \frac{\partial \mathcal{H}}{\partial q_{j}}+h_{j i} \frac{\partial \mathcal{H}}{\partial p_{j}}\right) \delta t,
\end{gathered}
$$

where we have used the fact that $h_{i j}=-h_{j i}$. An infinitesimal deformed phase space volume evolves with time through the relations (14) as

$$
d \tilde{\omega}\left(q^{\prime}, p^{\prime}\right)=\left|\frac{\partial\left(q_{i}^{\prime}, p_{i}^{\prime}\right)}{\partial\left(q_{i}, p_{i}\right)}\right| d \tilde{\omega}(q, p) .
$$

From relations (14) we have

$$
\begin{aligned}
& \frac{\partial q_{i}^{\prime}}{\partial q_{j}}=\delta_{i j}+\frac{\partial \delta q_{i}}{\partial q_{j}}, \quad \frac{\partial q_{i}^{\prime}}{\partial p_{j}}=\frac{\partial \delta q_{i}}{\partial p_{j}}, \\
& \frac{\partial p_{i}^{\prime}}{\partial q_{j}}=\frac{\partial \delta p_{i}}{\partial q_{j}}, \quad \frac{\partial p_{i}^{\prime}}{\partial p_{j}}=\delta_{i j}+\frac{\partial \delta p_{i}}{\partial p_{j}} .
\end{aligned}
$$

Using the above relations and up to the first order of $\delta t$ we have [18],

$$
\left|\frac{\partial\left(q_{i}^{\prime}, p_{i}^{\prime}\right)}{\partial\left(q_{i}, p_{i}\right)}\right|=1+\left(\frac{\partial \delta q_{i}}{\partial q_{i}}+\frac{\partial \delta p_{i}}{\partial p_{i}}\right) .
$$


Substituting this relation in the relation (16) one obtains

$$
\begin{aligned}
d \tilde{\omega}\left(q^{\prime}, p^{\prime}\right)=[1+ & \left(\frac{\partial f_{i j}}{\partial q_{i}}-\frac{\partial g_{j i}}{\partial p_{i}}\right) \frac{\partial \mathcal{H}}{\partial q_{j}} \delta t \\
& \left.+\left(\frac{\partial g_{i j}}{\partial q_{i}}-\frac{\partial h_{j i}}{\partial p_{i}}\right) \frac{\partial \mathcal{H}}{\partial p_{j}} \delta t\right] d \tilde{\omega}(q, p) .
\end{aligned}
$$

In the next step we should consider the time evolution of the Jacobian (9). For small deviation from the usual Poisson algebra, we have

$$
f_{i j} \ll 1, \quad\left(g_{i j}-\delta_{i j}\right) \ll 1, \quad h_{i j} \ll 1,
$$

independent of the explicit form of these functions. The above conditions ensure that the noncanonical sympelctic structure (1) reduces to the usual canonical ones in the low energy limit. In this limit, the Jacobian (9) can be approximated as [16]

$$
J(q, p)=\prod_{i=1}^{D}\left\{q_{i}, p_{i}\right\}=\prod_{i=1}^{D} g_{i i} \approx 1+\sum_{i=1}^{D}\left(g_{i i}-1\right) .
$$

The time evolution of the above Jacobian can be obtained through relations (14) as

$$
\begin{aligned}
J\left(q^{\prime}, p^{\prime}\right)=\prod_{i=1}^{D}\left\{q_{i}^{\prime}, p_{i}^{\prime}\right\}= & \prod_{i=1}^{D}\left(\left\{q_{i}, p_{i}\right\}+\left\{q_{i}, \delta p_{i}\right\}\right. \\
& \left.+\left\{\delta q_{i}, p_{i}\right\}+\left\{\delta q_{i}, \delta p_{i}\right\}\right) .
\end{aligned}
$$

The last term $\left\{\delta q_{i}, \delta p_{i}\right\}$ is the second order of $\delta t$ and can be ignored. Then, up to the first order of $\delta t$ we find

$$
J\left(q^{\prime}, p^{\prime}\right)=\prod_{i=1}^{D}\left\{q_{i}, p_{i}\right\}+\prod_{i=1}^{D}\left(\left\{q_{i}, \delta p_{i}\right\}+\left\{\delta q_{i}, p_{i}\right\}\right) .
$$

The first term in the right hand side of the above relation coincides with relation (21). Substituting from relations (15), to first order of $\delta t$ the relation (23) becomes

$$
\begin{aligned}
J\left(q^{\prime}, p^{\prime}\right)=J(q, p)+\prod_{i=1}^{D}[ & g_{i k}\left(\frac{\partial f_{k j}}{\partial q_{i}}-\frac{\partial g_{j i}}{\partial p_{k}}\right) \frac{\partial \mathcal{H}}{\partial q_{j}} \\
& +g_{i k}\left(\frac{\partial g_{k j}}{\partial q_{i}}-\frac{\partial h_{j i}}{\partial p_{k}}\right) \frac{\partial \mathcal{H}}{\partial p_{j}} \\
& -f_{i k}\left(\frac{\partial g_{i j}}{\partial q_{k}} \frac{\partial \mathcal{H}}{\partial q_{j}}+\frac{\partial h_{j i}}{\partial q_{k}} \frac{\partial \mathcal{H}}{\partial p_{j}}\right) \\
& \left.+h_{i k}\left(\frac{\partial f_{k j}}{\partial p_{i}} \frac{\partial \mathcal{H}}{\partial q_{j}}+\frac{\partial g_{j k}}{\partial p_{i}} \frac{\partial \mathcal{H}}{\partial p_{j}}\right)\right] \delta t,
\end{aligned}
$$

where we have used relation (21). In the light of the relation (20), one can neglect the second order terms

$$
\left(g_{i j}-\delta_{i j}\right) \times f_{i j}, \quad\left(g_{i j}-\delta_{i j}\right) \times h_{i j}, \quad f_{i j} \times h_{i j} .
$$

So, the last two terms can be neglected and the above Jacobian reduces to the following relation

$$
\begin{aligned}
J\left(q^{\prime}, p^{\prime}\right) \approx J(q, p)+ & \left(\frac{\partial f_{i j}}{\partial q_{i}}-\frac{\partial g_{j i}}{\partial p_{i}}\right) \frac{\partial \mathcal{H}}{\partial q_{j}} \delta t \\
& +\left(\frac{\partial g_{i j}}{\partial q_{i}}-\frac{\partial h_{j i}}{\partial p_{i}}\right) \frac{\partial \mathcal{H}}{\partial p_{j}} \delta t
\end{aligned}
$$

which after some manipulation becomes

$$
\begin{aligned}
\frac{J\left(q^{\prime}, p^{\prime}\right)}{J(q, p)}=1+J^{-1}(q, p) & {\left[\left(\frac{\partial f_{i j}}{\partial q_{i}}-\frac{\partial g_{j i}}{\partial p_{i}}\right) \frac{\partial \mathcal{H}}{\partial q_{j}}\right.} \\
+ & \left.\left(\frac{\partial g_{i j}}{\partial q_{i}}-\frac{\partial h_{j i}}{\partial p_{i}}\right) \frac{\partial \mathcal{H}}{\partial p_{j}}\right] \delta t .
\end{aligned}
$$

The inverse of the Jacobian can be approximated through the relation (21) as

$$
J^{-1}(q, p) \approx 1-\sum_{i=1}^{D}\left(g_{i i}-1\right),
$$

where we have used the relation (20). Substituting inverse of the Jacobian (27) and again neglecting the second order terms, one gets

$$
\begin{aligned}
\frac{J\left(q^{\prime}, p^{\prime}\right)}{J(q, p)}=1+ & \left(\frac{\partial f_{i j}}{\partial q_{i}}-\frac{\partial g_{j i}}{\partial p_{i}}\right) \frac{\partial \mathcal{H}}{\partial q_{j}} \delta t \\
& +\left(\frac{\partial g_{i j}}{\partial q_{i}}-\frac{\partial h_{j i}}{\partial p_{i}}\right) \frac{\partial \mathcal{H}}{\partial p_{j}} \delta t .
\end{aligned}
$$

From relations (28) and (19) we have

$$
\frac{d \tilde{\omega}\left(q^{\prime}, p^{\prime}\right)}{J\left(q^{\prime}, p^{\prime}\right)}=\frac{d \tilde{\omega}(q, p)}{J(q, p)},
$$

which ensures that the deformed phase space volume (11) is invariant under time evolution of the system and consequently the Liouville theorem is satisfied in the deformed phase space $\tilde{\Gamma}$. This result is very essential for our forthcoming arguments. We note that our general results obtained in this section include the results obtained in special cases studied previously [18, 19].

\section{THERMOSTATISTICS}

The volume of the phase space determines the number of microstates in the semiclassical regime and according to the Liuoville theorem it should be invariant under the time evolution. Now we are able to formulate the statistical mechanics in noncommutative phase space since the deformed density of states (10) is invariant under the time evolution through the relation (29). Moreover, one should also be careful about the definition of the bosons and fermions due to the loss of the Lorentz invariance in a noncommutative spacetime [25]. Here we suppose that fermions and bosons are defined in the same way as in the standard quantum mechanics but within the coherent state picture of noncommutativity which considers a particle as a smeared objects rather than to being a point-like particle. In other words, bosons and fermions save their quantum mechanical properties as in the standard quantum mechanics but the effect of noncommutativity of space is implemented by a substitution rule: the point-like structure of these particles is assumed to be replaced by a smeared, Gaussian profile. In this formalism, the particle mass $M$, instead of being completely 
localized at a point, is distributed throughout a region of linear size $\sqrt{\theta}$ (see part 2 of Appendix A). The implementation of this argument leads to the substitution of a position Dirac-delta function (which describes point-like structures) with a Gaussian profile describing smeared structures [7, 8, 26]. As has been shown in Ref. [27], the space noncommutativity enhances the negative statistical correlation between fermions and enhances the positive statistical correlation between bosons. Also, there are residual "attraction potential" between bosons and residual "repulsion potential" between fermions in the high temperature limit. So, in a noncommutative space the usual knowledge in statistical mechanics is still true, say the Bose-Einstein or Fermi-Dirac distributions with a modified density of states for smeared particles. With these points in mind, in which follows we treat the thermostatistics of bosons and fermions in this setup.

\section{A. The Method}

The issue of the noncommutativity can be included in the phase space by two equivalent pictures [28]: i) Working with the deformed commutation relation, such as (1), together with the non-deformed Hamiltonian function, ii) Finding canonical variables on the noncommutative phase space which satisfy the commutative algebra but the Hamiltonian function now gets modified to ensure that the Hamilton's equations (13) being the same in the two pictures. Mathematically, these two pictures are related to each other by the Darboux transformation. According to the Darboux theorem, it is always possible to find canonical coordinates on the symplectic manifold which satisfy commutative algebra. So, it is always possible to find a transformation that transforms any noncommutative Poisson algebra such as (11) to the commutative ones [29]. Of course, the Hamiltonian function gets modified when one transforms the noncommutative algebra to the commutative ones to ensure that the trajectories on the phase space remain the same in the two pictures. However, working within the first picture is more significant in statistical mechanics since in this picture noncommutativity only affects the number of microstates through the deformed density of states.

The number of particles $\mathrm{N}$ and pressure $\mathrm{P}$ of a statistical system with volume $V$ at temperature $T$ is given by the standard definitions

$$
\mathrm{N}=\sum_{\varepsilon}\left(z^{-1} e^{\varepsilon / T} \mp 1\right)^{-1},
$$

and

$$
\mathrm{P} V=\mp T \sum_{\varepsilon} \ln \left(1 \mp z e^{-\varepsilon / T}\right),
$$

respectively, where $z$ is the fugacity of the system and signs $(-)$ and $(+)$ hold for bosons and fermions respectively. The energy of the microstates $\varepsilon$ should be determined only by quantized theory. In usual statistical mechanics, $\varepsilon$ is the solution of the Schrödinger equation. Here it should be a solution of the full quantum gravity equations for the corresponding statistical system. But, one can replace summation over $\varepsilon$ by the integral over all phase space variables by means of the density of states (10) as $\sum_{\varepsilon} \rightarrow \frac{1}{(2 \pi)^{3}} \int_{V} \int \frac{d^{3} q d^{3} p}{J(q, p)}$, where $V$ is the volume of the corresponding statistical system. In the early Universe, all the particles effectively are relativistic and the Hamiltonian simplifies to $\mathcal{H}(p)=p$ (where $p$ is the norm of the vector $p_{i}$ ). We set also $z=1$ (the chemical potential to be zero) as usually one assumes. Now, the number of particles and pressure in quantum gravity regime can be obtained from the relations (30) and (31) as

$$
\begin{gathered}
\mathrm{N}_{\mp}=\frac{g_{\mp}}{(2 \pi)^{3}} \int_{V} \int\left(e^{p / T} \mp 1\right)^{-1} \frac{d^{3} q d^{3} p}{J(q, p)}, \\
\mathrm{P}_{\mp}=\mp \frac{g_{\mp}}{(2 \pi)^{3}} \frac{T}{V} \int_{V} \int \ln \left(1 \mp e^{-p / T}\right) \frac{d^{3} q d^{3} p}{J(q, p)},
\end{gathered}
$$

where $g_{-}$and $g_{+}$are the number of relativistic degrees of freedom for bosons and fermions respectively. In the above relations, the sign $(-)$ and $(+)$ hold for the bosons and fermions respectively. The usual results can be recovered by setting $J=1$ which is corresponding to the identity transformation with $q=Q$ and $p=P$ in relation (77). The total number of particles and total pressure is given by

$$
\mathrm{N}=\mathrm{N}_{-}+\mathrm{N}_{+}
$$

and

$$
\mathrm{P}=\mathrm{P}_{-}+\mathrm{P}_{+} .
$$

The entropy density $s$ and the energy density $\rho$ of the system can be obtained from the definitions

$$
s(T)=\frac{\partial \mathrm{P}}{\partial T}, \quad \rho(T)=T^{2} \frac{\partial}{\partial T}\left(\frac{\mathrm{P}}{T}\right) .
$$

Now, the semiclassical statistical consideration is completed and one can obtain any thermodynamical quantities in noncommutative phase spaces through the relations (34), (35), and (36).

\section{B. Cosmological Implications}

Before considering particular examples of the noncommutative phase space, we study some implications of our setup on the thermodynamics of the early Universe.

The dynamics of the Universe in the standard cosmology is given by the Einstein equations, the so-called Friedmann and Raychaudhuri equations,

$$
\begin{aligned}
& \left(\frac{\dot{a}}{a}\right)^{2}+\frac{k}{a^{2}}=\frac{8 \pi G}{3} \rho, \\
& \frac{\ddot{a}}{a}=-\frac{4 \pi G}{3}(\rho+3 \mathrm{P}),
\end{aligned}
$$


where $a(t)$ is the scale factor, $\rho$ and $\mathrm{P}$ are the energy density and pressure respectively, and a dot denotes derivative with respect to the cosmic time. Here $k$ marks the spatial curvature which is normalized to zero, 1 and -1 for flat, closed and open Universes, respectively. Furthermore, an equation of state should be supplemented to complete this set of equations. In fact, an equation of state parameter of the form $\mathrm{P}=\mathrm{P}(\rho, s)$ determines whether the Universe is accelerating or decelerating, through the Raychaudhuri equation. In principle, the equation of state should be obtained from the statistical considerations of the particles in the early Universe. So, the question is that whether equation of state remains unchanged in the limit of high temperature? From the relation (33) and the definition (36) for the energy density, it is clear that the equation of state changes when one includes quantum gravity effects. We find such a modification to the equation of state in Snyder spaces in the next section.

Furthermore, it is also important to note that the entropy density (36) now changes since the pressure is modified through the relation (33). Consequently, the adiabatic condition

$$
S=s a^{3}=\text { cte. }
$$

where $S$ is the total entropy of the Universe, gets modified in quantum gravity regime. Such a modification to the entropy density removes the Big Bang singularity in a fascinating manner. We will see this feature explicitly in the case of Snyder noncommutative space in the next section.

\section{THE SNYDER UNIVERSE}

The Snyder noncommutative spacetime was firstly introduced by Snyder [9]. The corresponding noncommutative phase space has recently been developed in Ref. [10] by means of an appropriate structure with the following noncommutative commutation relations (as has been shown by Mignemi in [10], this is actually the Snyder space on a sphere)

$$
\begin{array}{r}
\left\{q_{i}, q_{j}\right\}=\beta^{2} J_{i j}, \quad\left\{p_{i}, p_{j}\right\}=\alpha^{2} J_{i j}, \\
\left\{q_{i}, p_{j}\right\}=\delta_{i j}+\alpha^{2} q_{i} q_{j}+\beta^{2} p_{i} p_{j}+2 \alpha \beta p_{i} q_{j},
\end{array}
$$

where $i, j=1,2, \ldots, D$ and $J_{i j}=q_{i} p_{j}-q_{j} p_{i}$ are the generators of the rotation in $D$ dimensions. The deformation parameters $\alpha$ and $\beta$ induce the IR and UV cutoffs respectively. We need the Jacobian (9) corresponding to the Snyder algebra (40) to study the thermodynamics in this framework by using the relations (34), (35), and (36). In Appendix A we have calculated the Jacobian for the Snyder space which is

$$
J(q, p)=1+3|\alpha \mathbf{q}+\beta \mathbf{p}|^{2} .
$$

where we have set $D=3$ for a single-particle states and $\mathbf{q}$ and $\mathbf{p}$ are the 3 -vectors associated to the $q_{i}$ and $p_{i}$ respectively. In Appendix $\mathrm{A}$ we have shown that the Jacobian (41) also supports the other approaches to the noncommutativity such as the coherent state approach [7, 8]. The Jacobian (41) contains the UV/IR mixing effect which is a common feature of the noncommutative spaces. Both the IR and UV cutoffs are essential for the renormalization of the quantum fields in curved spaces. Substituting the Jacobian (41) in the relation (33) gives the pressure for the bosons and fermions as

$$
\begin{gathered}
\mathrm{P}_{\mp}=\mp \frac{g_{\mp}}{(2 \pi)^{3}} \frac{T}{V} \int_{V} \int \ln \left(1 \mp e^{-p / T}\right) \times\left(1-3 \alpha^{2} q^{2}-3 \beta^{2} p^{2}-6 \alpha \beta \mathbf{q} \cdot \mathbf{p}\right) d^{3} q d^{3} p \\
=\mp \frac{g_{\mp}}{(2 \pi)^{3}} \frac{T}{V}\left(\int_{V} d^{3} q \times \int \ln \left(1 \mp e^{-p / T}\right) d^{3} p-3 \alpha^{2} \int_{V} q^{2} d^{3} q \times \int \ln \left(1 \mp e^{-p / T}\right) d^{3} p\right. \\
\left.-3 \beta^{2} \int_{V} d^{3} q \times \int \ln \left(1 \mp e^{-p / T}\right) p^{2} d^{3} p-6 \alpha \beta \int_{V} \int \mathbf{q} \cdot \mathbf{p} \ln \left(1 \mp e^{-p / T}\right) d^{3} q d^{3} p\right),
\end{gathered}
$$

where we have expanded the Jacobian up to the second order of the deformation parameters $\alpha$ and $\beta$. Only the last term in the right hand side of the above relation includes both of the deformation parameters $\alpha$ and $\beta$. Now we calculate this UV/IR mixing term.
Writing the 3 -vectors $\mathbf{q}$ and $\mathbf{p}$ in the spherical coordinates as $\mathbf{q}=\left(q, \theta_{1}, \varphi_{1}\right)$ and $\mathbf{p}=\left(p, \theta_{2}, \varphi_{2}\right)$ with $q=|\mathbf{q}|$ and $p=|\mathbf{p}|$, the inner product will be $\mathbf{q} \cdot \mathbf{p}=$ $q p\left(\cos \theta_{1} \cos \theta_{2}+\sin \theta_{1} \sin \theta_{2} \cos \left(\varphi_{1}-\varphi_{2}\right)\right)$ and the last term in the relation (42) becomes 


$$
\begin{aligned}
-6 \alpha \beta \int_{V} \int \mathbf{q} \cdot \mathbf{p} & \ln \left(1 \mp e^{-p / T}\right) d^{3} q d^{3} p=-6 \alpha \beta \int_{0}^{\infty} d p p^{3} \ln \left(1 \mp e^{-p / T}\right) \int_{-1}^{+1} d\left(\cos \theta_{2}\right) \int_{0}^{2 \pi} d \varphi_{2} \\
\times & \int_{0}^{R} d q q^{3} \int_{-1}^{+1} d\left(\cos \theta_{1}\right) \int_{0}^{2 \pi} d \varphi_{1}\left(\cos \theta_{1} \cos \theta_{2}+\sin \theta_{1} \sin \theta_{2} \cos \left(\varphi_{1}-\varphi_{2}\right)\right)=0
\end{aligned}
$$

where we have used the integrals $\int_{-1}^{+1} d\left(\cos \theta_{1}\right) \cos \theta_{1}=0$ and $\int_{0}^{2 \pi} d \varphi_{1} \cos \left(\varphi_{1}-\varphi_{2}\right)=0$. So, the last term in the relation (42) vanishes. The integral over coordinates simply gives $\int_{V} d^{3} q=V, \int_{V} q^{2} d^{3} q=\frac{4 \pi}{5} R^{5}=\frac{3}{5}\left(\frac{3}{4 \pi}\right)^{2 / 3} V^{5 / 3}$ where $R$ is the radius corresponding to the volume of the system under consideration. Performing the integrals over momentums by using the identity $\int_{0}^{\infty} \frac{x^{n-1} d x}{e^{x}+1}=$ $\left(1-\frac{1}{2^{n-1}}\right) \int_{0}^{\infty} \frac{x^{n-1} d x}{e^{x}-1}$ gives the pressure for the bosons and fermions respectively as

$$
\begin{aligned}
& \mathrm{P}_{-}=g_{-} \frac{\pi^{2} T^{4}}{90}\left(1-\frac{9}{5}\left(\frac{3}{4 \pi}\right)^{\frac{2}{3}} \alpha^{2} V^{\frac{2}{3}}-\frac{24}{7} \beta^{2} \pi^{2} T^{2}\right),(44) \\
& \mathrm{P}_{+}=\frac{7}{8} g_{+} \frac{\pi^{2} T^{4}}{90}\left(1-\frac{9}{5}\left(\frac{3}{4 \pi}\right)^{\frac{2}{3}} \alpha^{2} V^{\frac{2}{3}}-\frac{186}{49} \beta^{2} \pi^{2} T^{2}\right),
\end{aligned}
$$

where as usual $g_{-}$and $g_{+}$are the number of relativistic degrees of freedom for bosons and fermions respectively. The natural choice for the IR deformation parameter is the square root of the cosmological constant with $\alpha \sim$ $10^{-24} \mathrm{~cm}^{-1}$, and for the UV deformation parameter $\beta=$ $\beta_{0} l_{P}=\beta_{0} T_{P}^{-1}$ is relevant to ensure that the UV effects only become important around the Planck scale. The numerical constant $\beta_{0} \sim \mathcal{O}(1)$ should be fixed only with experiment [32]. The total pressure of the system can be obtained from the relations (35) and (44) as

$$
\mathrm{P}(T)=g_{\star} \frac{\pi^{2} T^{4}}{90}\left(1-\frac{9}{5}\left(\frac{3}{4 \pi}\right)^{\frac{2}{3}} \alpha^{2} V^{\frac{2}{3}}-\chi\left(T / T_{P}\right)^{2}\right),
$$

where $g_{\star}=\left(g_{-}+\frac{7}{8} g_{+}\right)$and $\chi=\frac{24 \pi^{2}}{7}\left(\frac{g_{-}+\frac{31}{32} g_{+}}{g_{-}+\frac{7}{8} g_{+}}\right) \beta_{0}^{2}$. We are interested in the early Universe implications and the IR term in the relation (45) is negligible in the high temperature limit. Nevertheless, the very small IR effects play an essential role for the renormalization of the quantum fields in the curved space. Therefore, the total pressure in the high temperature limit will be

$$
\mathrm{P}(T)=g_{\star} \frac{\pi^{2} T^{4}}{90}\left(1-\chi\left(T / T_{P}\right)^{2}\right) .
$$

The corresponding energy density can be obtained through the definition (36) as

$$
\rho(T)=g_{\star} \frac{\pi^{2} T^{4}}{30}\left(1-\frac{5}{3} \chi\left(T / T_{P}\right)^{2}\right) .
$$

\section{A. Energy Conditions and Equation of State Parameter}

The results (46) and (47) show that the pressure and energy density are not always positive definite in Snyder

\begin{tabular}{|c|c|}
\hline Energy conditions & Temperature \\
\hline DEC, WEC, SEC & $0<T<\frac{1}{\sqrt{2 \chi}} T_{P}$ \\
\hline WEC, SEC & $0<T<\sqrt{\frac{3}{5 \chi}} T_{P}$ \\
\hline SEC & $0<T<\sqrt{\frac{2}{3 \chi}} T_{P}$ \\
\hline$\rho<0, \quad \mathrm{P} \leq 0$ & $T \geq \frac{1}{\sqrt{\chi}} T_{P}$ \\
\hline
\end{tabular}

TABLE I. Energy conditions in Snyder noncommutative space

space. So one can not use these results in the Einstein equations (37) and (38) without considering the energy conditions for them. While both of the pressure and energy density get negative values in the limit of high temperature $T \rightarrow \infty$, the energy conditions give a correct picture for these thermodynamical quantities. In table II we represent the temperature intervals for the validity of the Dominant Energy Condition (DEC), Weak Energy Condition (WEC), and Strong Energy Condition (SEC). The pressure always is positive for the temperature $T<\frac{1}{\sqrt{\chi}}$, so all of the intervals in the table $\square$ are the subset of the domain of validity of DEC, WEC, and SEC. In what follows, we consider the thermodynamics of the Snyder space in different energy conditions separately.

The energy density as a function of the temperature is shown in figure 1. Interestingly, the infinite energy density which appears in the standard Big Bang model now is removed and the energy density behaves differently in the high energy regime. We will show that this result emerges because the Big Bang singularity is removed in the Snyder space.

The equation of state parameter $w=\mathrm{P} / \rho$ becomes

$$
w(T)=\frac{1-\chi\left(T / T_{P}\right)^{2}}{3-5 \chi\left(T / T_{P}\right)^{2}} .
$$

The above relation correctly reduces to the usual radiation dominated case in the limit of low temperatures $w(T \rightarrow 0)=\frac{1}{3}$. This form of the equation of state is very similar to the one obtained in Ref. [31], where the authors proposed a noncommutative inflation in the framework of the varying speed of light theories. Clearly, the condition $w=-1$ is now possible for the temperature $T=\sqrt{\frac{2}{3 \chi}} T_{P}$ where only the SEC is satisfied. While the SEC is satisfied in this temperature, the energy density (47) becomes negative for this temperature (see also figure 11). In this situation the condition $w=-1$ doesn't give accelerating expansion since gravity is always attractive when the SEC is satisfied. The temperature evolution of the equation of state parameter is shown in figure 2. The equation of state parameter varies in the range of 


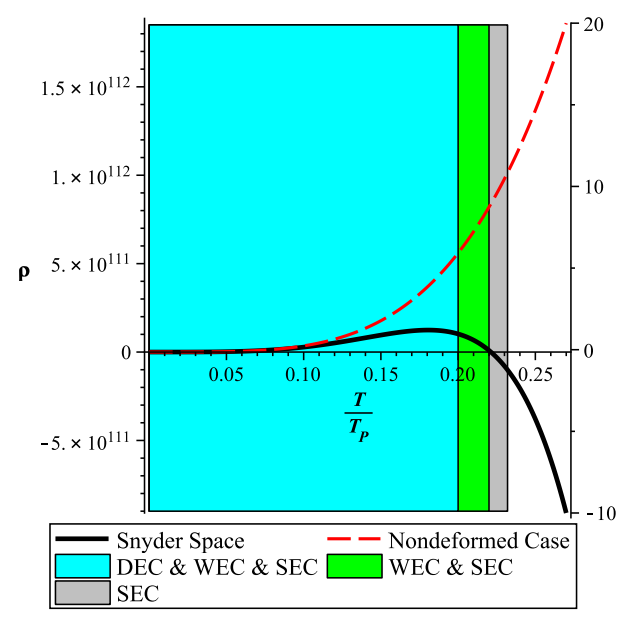

FIG. 1. The energy density versus the temperature in the Snyder space. The deformation parameter is taken to be $\beta_{0}=1$ and the number of relativistic degrees of freedom for the bosons and fermions are taken as $g_{-}=2$ and $g_{+}=10$ respectively. In the limit of high temperature, the quantum gravity effects become efficient and the energy density decreases and consequently the infinite energy density in the Big Bang model disappears. The domain of the validity of the energy conditions are shown with cyan, green and silver colors.

$\frac{1}{3} \leq w \leq 1$ when all of the DEC, WEC, and SEC simultaneously are satisfied. It varies in the range $w \in[1,+\infty)$ when both the WEC and SEC are satisfied. The negative values for the equation of state parameter $w \in(-\infty,-1]$ are allowed when only the SEC is satisfied.

\section{B. Entropy Density and Big Bang Singularity}

As we have seen previously, the entropy density modifies when one considers the quantum gravity effects in the thermodynamics of the early Universe through the relations (33) and (36). This different entropy density significantly changes the adiabatic condition (39) which determines the temperature evolution of the Universe. The entropy density in high temperature limit can be obtained by substituting the relation (46) in (36)

$$
s(T)=g_{\star} \frac{2 \pi^{2} T^{3}}{45}\left(1-\frac{3}{2} \chi\left(T / T_{P}\right)^{2}\right) .
$$

Note that for $w=-1$ we find $s\left(\sqrt{\frac{2}{3 \chi}} T_{P}\right)=0$ since definitions (36) indicate that $s=\frac{\rho+\mathrm{P}}{T}$. The temperature behavior of the entropy density is shown in figure 3 .

An important point here is that at the very high temperature, the quantum gravitational effects dominate and the modified entropy density (49) changes significantly the adiabatic condition (39). In the usual radiation dominated era, the entropy density is proportional to the temperature as $\propto T^{3}$. As the temperature increases (toward

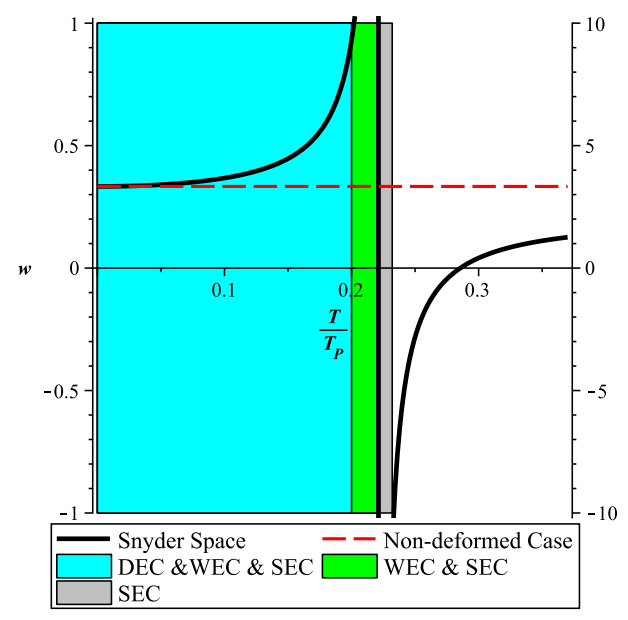

FIG. 2. The equation of state parameter $w$ which becomes a function of temperature in the Snyder space. In the cyan region, all of the DEC, WEC, and SEC energy conditions are satisfied and the equation of state parameter is restricted to the range $\frac{1}{3} \leq w \leq 1$. In the green region, where WEC and SEC are satisfied, $w \in[1,+\infty)$. In the silver region when only the $\mathrm{SEC}$ is satisfied, $w \in(-\infty,-1]$. In this region the condition $w=-1$ is possible, however this holds for negative values of the energy density and therefore doesn't give an accelerating expansion.

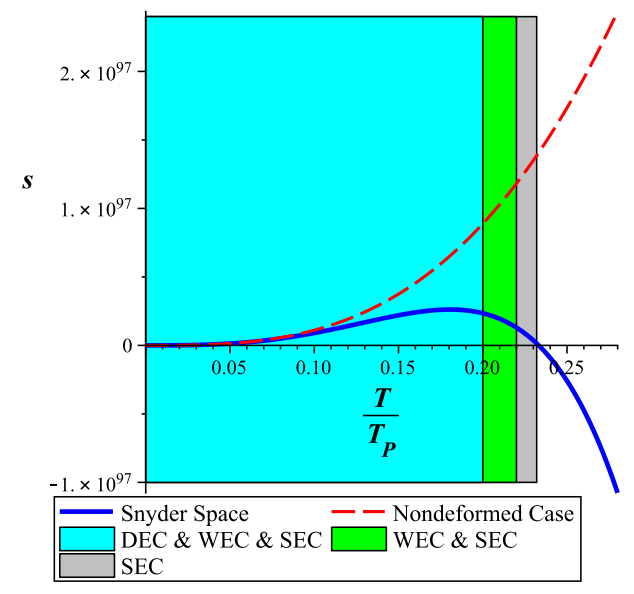

FIG. 3. Entropy density variation versus the temperature in the Snyder space. The unusual behavior of the entropy density simultaneously resolves the large entropy density problem and the Big Bang singularity.

the singularity of the standard model), the usual (nondeformed) entropy density increases and consequently the scale factor should be reduced to respect the adiabatic condition (39). But, the entropy density (49) behaves very differently in the high temperature limit. As temperature approaches the Planck temperature, the second term on the right hand side of the relation (49) dominates and consequently the entropy density decreases instead of increasing (see figure 33). Interestingly, the large en- 


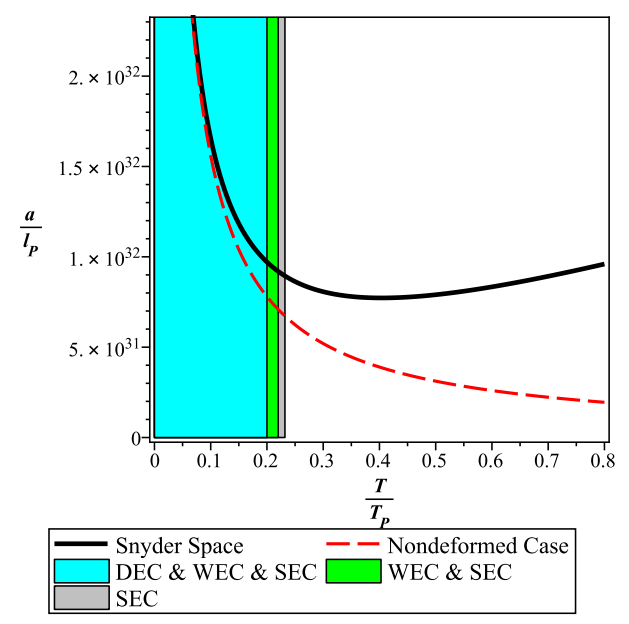

FIG. 4. The scale factor versus the temperature in the Snyder space. The scale factor has a nonzero minimum size which leads to a nonsingular Universe.

tropy density problem is resolved in this way. On the other hand, the scale factor can be obtained from the adiabatic condition (39) as

$$
a(T) \approx \frac{\vartheta}{T}\left(1+\frac{\chi}{2}\left(T / T_{P}\right)^{2}\right),
$$

where $\vartheta^{3}=\frac{45 S}{2 \pi^{2}\left(g_{-}+\frac{7}{8} g_{+}\right)}$is a numerical constant. The behavior of the scale factor as a function of temperature is shown in figure 4 .

The scale factor in the standard radiation dominated era can be obtained by setting $\chi$ to be zero in relation (50) as $a(T)=\frac{\vartheta}{T}$ which is shown in figure 4 by red dashed line. This scale factor has a singularity at the early time or equivalently at the very high temperature. However, the scale factor (50) has a nonzero minimum size $a=(\sqrt{2 \chi} \vartheta) l_{P}$ at the temperature $T=\sqrt{\frac{2}{\chi}} T_{P}$. From the table \it is clear that this minimum occurs in the region that non of the energy conditions are satisfied. The minimum size for the universe when at least the WEC is satisfied happens for temperature $T=\sqrt{\frac{3}{5 \chi}} T_{P}$ and is given by

$$
a_{\min }=\left(\frac{13}{2} \sqrt{\frac{\chi}{15}} \vartheta\right) l_{P} .
$$

So, the Big Bang singularity is removed in this setup.

\section{CONCLUSIONS}

The Universe comes from the singularity when one uses the classical general relativity equations to describe the cosmic evolution. It is natural to expect that the Big Bang singularity will be removed when one uses the yet unknown full quantum gravitational equations. While there is no full quantum gravity theory today, some candidates such as the string theory and loop quantum gravity revealed some aspects of the ultimate quantum gravity theory. The minimal measurable length and minimal momentum, which induce respectively the UV and IR cutoffs in the corresponding theory, are the common addresses of all promising candidates of quantum gravity proposal. In this paper we firstly constructed a general deformed phase space with UV and IR cutoffs by means of the deformed commutation relations. We have shown that the Liouville theorem is satisfied in this framework which ensures that the number of microstates remains unchanged under the time evolution in the deformed phase space. Then we formulated a general statistical physics which contains the UV and IR cutoffs. We have studied the effects of the noncommutativity on the energy density and pressure of the statistical system in the Snyder space and we have treated the energy conditions in this framework since now $\rho$ and $\mathrm{P}$ are not always positive definite. We have shown that the equation of state parameter is temperature dependent in the UV sector of the theory which opens a possible window for the natural realization of the inflation in this setup. The energy density of the Universe becomes finite when the DEC and WEC are satisfied and the condition $\mathrm{P}=-\rho$ is possible in the SEC, however this gives no accelerated expansion since gravity is always attractive when SEC is satisfied. Furthermore, the adiabatic condition $s a^{3}=$ cte changes in this setup since the entropy density gets modified when one considers quantum gravity effects. While the usual entropy density always increases as the temperature increases, the deformed entropy density behaves very differently in the Snyder space. The modified entropy density decreases with temperature in the high energy regime and the adiabatic condition implies that the scale factor gets a minimum size as $a \propto l_{P}$, where $l_{P}$ is the Planck length. Consequently, the Big Bang singularity will be removed in this framework. As the final remark, we note that Einstein's equations should be modified in the high energy regime where the noncommutative effects are important. Some attempts have been made in this direction (see for instance [7, 33]), but no complete noncommutative general relativity have been formulated yet.

\section{Appendix A: Jacobian in Noncommutative Spaces}

In this appendix, we consider three different approaches to the issue of the noncommutative geometry; Snyder noncommutative spaces, coherent state approach, and the Moyal product law.

\section{Snyder Spaces}

We calculate the Jacobian (91) for the Snyder algebra (40) in this section. For the small deviation from the canonical Poisson algebra (20), equivalent to $\beta, \alpha \rightarrow 0$ 
in relation (40), one can use the approximate relation (21) (see Appendix of the Ref. [16]). In this case, the corresponding Jacobian becomes

$$
\begin{array}{r}
J(q, p)=\underbrace{\left(1+|\alpha \mathbf{q}+\beta \mathbf{p}|^{2}\right) \times \ldots \times\left(1+|\alpha \mathbf{q}+\beta \mathbf{p}|^{2}\right)}_{D \text { times }} \\
=\left(1+|\alpha \mathbf{q}+\beta \mathbf{p}|^{2}\right)^{D} \cdot(\mathrm{A}-1)
\end{array}
$$

where $\mathbf{q}$ and $\mathbf{p}$ are the $D$-vector associated to the $q_{i}$ and $p_{i}$ respectively. Expanding above relation in the limit of $\alpha, \beta \rightarrow 0$, up to quadratic order of deformation parameters, the Jacobian becomes

$$
J(q, p)=1+D|\alpha \mathbf{q}+\beta \mathbf{p}|^{2} .
$$

Having Jacobian in hand, one can study the thermodynamics of the system through the relations (32) and (33).

\section{The Coherent States Approach}

The coherent state approach to the noncommutative geometry was extended in Ref. 7] by means of the kernels in Feynman path integral approach in quantum field theory (see also [30]) . The noncommutativity affects the Feynman propagator in momentum space as

$$
G_{\theta}\left(p^{2} ; m^{2}\right)=\frac{1}{(2 \pi)^{D}} \frac{e^{-\theta p^{2}}}{p^{2}+m^{2}},
$$

where $\theta$ is the noncommutativity parameter and $p^{2}=$ $p_{1}^{2}+p_{2}^{2}+\ldots+p_{D}^{2}$. The exponential term in the above relation induces a UV cutoff in the high energy regime. A more general case was extended in Ref. [8] that includes an IR cutoff as well as UV cutoff. They considered a toy model in which the IR and UV terms appears as an exponential term $e^{-\sigma q^{2}-\theta p^{2}}$, where $\sigma$ is another deformation parameter that induces an IR cutoff and $q^{2}=q_{1}^{2}+q_{2}^{2}+\ldots+q_{D}^{2}$. This exponential term is effectively equivalent to the inverse of the Jacobian in our study as

$$
J^{-1}=e^{-\sigma q^{2}-\theta p^{2}} .
$$

Expanding the above relation in the limit of $\sigma, \theta \rightarrow 0$, up to the first order of the deformation parameters gives

$$
J(p)=1+\sigma q^{2}+\theta p^{2}+\mathcal{O}\left(\sigma^{2}, \theta^{2}\right),
$$

which is clearly equivalent to the Snyder case (A-2) by identifying $\sigma=D \alpha$ and $\theta=D \beta^{2}$. However, the Jacobian (A-2) include an extra mixing term which doesn't affect thermodynamics of the early Universe through the relation (42).

\section{Moyal Product Law}

The last approach to the noncommutative phase space is described by the star product, known as the Moyal product law, between two arbitrary functions of position and momentum as 34]

$$
\left(f *_{\alpha} g\right)(x)=\left.\exp \left(\frac{1}{2} \alpha^{a b} \partial_{a}^{(1)} \partial_{b}^{(2)}\right) f\left(x_{1}\right) g\left(x_{2}\right)\right|_{x_{1}=x_{2}=x}
$$

such that

$$
\alpha_{a b}=\left(\begin{array}{cc}
\theta_{i j} & \delta_{i j}+\sigma_{i j} \\
-\delta_{i j}-\sigma_{i j} & \beta_{i j}
\end{array}\right)
$$

where the $D \times D$ matrices $\theta$ and $\beta$ are assumed to be antisymmetric with $2 D$ being the dimension of the classical phase space, representing the noncommutativity in coordinates and momenta, respectively. In contrast to the Poisson brackets, the Moyal brackets can be written as

$$
\{f, g\}_{\alpha}=f *_{\alpha} g-g *_{\alpha} f .
$$

A simple calculations shows that

$\left\{q_{i}, q_{j}\right\}_{\alpha}=\theta_{i j}, \quad\left\{q_{i}, p_{j}\right\}_{\alpha}=\delta_{i j}+\sigma_{i j}, \quad\left\{p_{i}, p_{j}\right\}_{\alpha}=\beta_{i j}$.

Now, consider the transformation (7) in the classical phase space as

$\left(Q_{i}, P_{i}\right) \rightarrow\left(q_{i}=Q_{i}-\frac{1}{2} \theta_{i j} P_{j}, \quad p_{i}=P_{i}+\frac{1}{2} \beta_{i j} Q_{j}\right)$.

It is easy to show that the new phase space variables $q_{i}$ and $p_{i}$ satisfy

$\left\{q_{i}, q_{j}\right\}=\theta_{i j}, \quad\left\{q_{i}, p_{j}\right\}=\delta_{i j}+\sigma_{i j}, \quad\left\{p_{i}, p_{j}\right\}=\beta_{i j},(\mathrm{~A}-11)$ with $\sigma_{i j}=-\frac{1}{8}\left(\theta_{i}^{k} \beta_{k j}+\beta_{i}^{k} \theta_{k j}\right)$. The commutation relations (A-11) are the same as A-9). So, transformation (7) transforms commutative phase space to the Moyal noncommutative ones. It is more convenient to work with Poisson brackets (A-11) than $\alpha$-star Moyal brackets (A-9). It is important to note that the relations (A-9) are defined in the spirit of the Moyal product given above. However, in the relations defined by (A-11), the new phase space variables $q_{i}$ and $p_{i}$ are functions of $Q_{i}$ and $P_{i}$ which obey the usual Poisson bracket relations (8). So the relations (A-9) and (A-11) should be considered as distinct.

Now, we need only the Jacobian of this transformation for our purpose. For small deviation from the Poisson algebra $\left(\theta_{i j}=\beta_{i j}=\sigma_{i j} \approx 0\right)$, the relation (21) is a good approximation which gives the result

$$
J=1+\sigma_{11}+\ldots+\sigma_{D D}=1+\operatorname{Tr}(\sigma) .
$$

Note that the Jacobian becomes constant since the deformation parameters $\theta_{i j}, \beta_{i j}$ and $\sigma_{i j}$ are constant. Consequently, the Moyal noncommutativity gives no significant modification to the thermodynamical quantities through relations (32) and (33) since it multiplies the equations just by a constant numerical factor.

\section{Acknowledgement}

We would like to thank two anonymous referees for very insightful comments. 
[1] W. Rindler, Gen. Rel. Grav. 34 (2002) 133 C. B. Collins and S. W. Hawking, Astrophys. J. 180 (1973) 317.

[2] A. H. Guth, Phys. Rev. D 23 (1981) 347.

[3] A. D. Linde, Phys. Lett. B 108 (1982) 389 A. Albrecht and P. J. Steinhardt, Phys. Rev. Lett. 48 (1982) 1220.

[4] D. J. Gross and P. F. Mende, Nucl. Phys. B 303 (1988) 407

D. Amati, M. Ciafaloni and G. Veneziano, Phys. Lett. B 216 (1989) 41

M. Maggiore, Phys. Lett. B 319 (1993) 83

L. Garay, Int. J. Mod. Phys. A 10 (1995) 145.

[5] A. Kempf, G. Mangano and R. B. Mann, Phys. Rev. D 52 (1995) 1108

A. Kempf and G. Mangano, Phys. Rev. D 55 (1997) 7909 K. Nozari and A. Etemadi, Phys. Rev. D 85 (2012) 104029.

[6] G. A. Camelia, Int. J. Mod. Phys. D 11 (2000) 35

G. A. Camelia, Nature 418 (2002) 34

J. Magueijo and L. Smolin, Phys. Rev. Lett. 88 (2002) 190403.

[7] A. Smailagic and E. Spallucci, J. Phys. A 36 (2003) L467 A. Smailagic and E. Spallucci, J. Phys. A 36 (2003) L517.

[8] M. Kober and P. Nicolini, Class. Quantum Grav. 27 (2010) 245024.

[9] H. Snyder, Phys. Rev. 71 (1947) 38.

[10] S. Mignemi, Phys. Rev. D 84 (2011) 025021 S. Mignemi, Class. Quantum Grav. 29 (2012) 215019.

[11] A. Ashtekar, S. Fairhurst and J. Willis, Class. Quantum Grav. 20 (2003) 1031

A. Corichi, T. Vukašinac and J. A. Zapata, Phys. Rev. D 76 (2007) 0440163.

[12] A. Corichi, T. Vukašinac and J.A. Zapata, Phys. Rev. D 76 (2007) 044016.

[13] A. Corichi and T. Vukašinac, Phys. Rev. D 86 (2012) 064019.

[14] G. M. Hossain, V. Husain and S. S. Seahra, Class. Quantum Grav. 27 (2010) 165013

B. Majumder and S. Sen, Phys. Lett. B 717 (2012) 291.

[15] We work in natural units $\hbar=c=k_{B}=1$, where $\hbar, c$ and $k_{B}$ are the Planck constant, speed of light in vacuum and Boltzman constant, respectively.

[16] T. Fityo, Phys. Lett. A 372 (2008) 5872.

[17] B. Vakili and M. A. Gorji, J. Stat. Mech. (2012) P10013.

[18] L. N. Chang, D. Minic, N. Okamura and T. Takeuchi, Phys. Rev. D 65 (2002) 125028.

[19] A. F. Ali, Class. Quantum Grav. 28 (2011) 065013

P. Pedram, Phys. Lett. B 718 (2012) 638

S. Jalalzadeh, M. A. Gorji and K. Nozari, Gen. Rel. Grav. 46 (2014) 1632.

[20] M. Lubo, Phys. Rev. D 68 (2003) 125004
P. Wang, H. Yang and X. Zhang, JHEP 08 (2010) 043 P. Wang, H. Yang and X. Zhang, Phys. Lett. B 718 (2012) 265.

[21] S. Kalyana Rama Phys. Lett. B 519 (2001) 103 M. Lubo, hep-th/0009162

[22] G. Amelino-Camelia and S. Majid, Int. J. Mod. Phys. A 15 (2000) 4301

J. Kowalski-Glikman, Phys. Lett. A 299 (2002) 454

J. C. Niemeyer, Phys. Rev. D 65 (2002) 083505

S. Das and D. Roychowdhury, Phys. Rev. D 81 (2010) 085039

G. Amelino-Camelia, N. Loret, G. Mandanici and F. Mercati, Int. J. Mod. Phys. D 21 (2012) 1250052

G. Amelino-Camelia, M. Arzano, G. Gubitosi and J. Magueijo, Phys. Rev. D 87 (2013) 123532.

[23] X. Zhang, L. Shao and Bo-Qiang Ma, Astropart. Phys. 34 (2011) 840.

[24] J. Fernando Barbero G., J. Prieto and E. J. S. Villaseor, Class. Quantum Grav. 30 (2013) 165011

M. A. Gorji, K. Nozari and B. Vakili, arXiv:1312.6835.

[25] N. Seiberg, L. Susskind, and N. Toumbas, JHEP 0006 (2000) 044

S. M. Carroll, J. A. Harvey, V. A. Kostelecky, C. D. Lane, T. Okamoto Phys. Rev. Lett. 87 (2001) 141601.

[26] K. Nozari and S. H. Mehdipour, Class. Quantum Grav. 25 (2008) 175015

K. Nozari and S. H. Mehdipour, JHEP 0903 (2009) 061.

[27] W. -H. Huang and K. -W. Huang, Phys. Lett. B 670 (2009) 416.

[28] Also, there is another approach to the issue, the so-called coherent states approach, which directly deformes the density of states on the phase space. However, this approach can be effectively realized from the deformed commutation relation (1) in the light of the Jacobian (9) and the corresponding deformed density of states (10).

[29] V.I. Arnold, Mathematical Methods of Classical Mechanics, Springer-Verlag, New York, 1989.

[30] H. Perrier, R. Durrer and M. Rinaldi, JHEP 01 (2013) 067.

[31] S. Alexander, R. Brandenberger and J. Magueijo, Phys. Rev. D 67 (2003) 081301

S. Alexander and J. Magueijo, hep-th/0104093.

[32] S. Das and E. C. Vagenas, Phys. Rev. Lett. 101 (2008) 221301

P. Pedram, K. Nozari and S. H. Taheri, JHEP 1103 (2011) 093.

[33] R. J. Szabo, Class. Quantum Grav. 23 (2006) R199.

[34] J. E. Moyal, Proc. Camb. Phil. Soc. 45 (1949) 99 M. Przanowski and J. Tosiek, Acta Phys. Pol. B 30 (1999) 179

J. M. Romero, J. A. Satiago, and D. Vergara, Phys. Lett. A 310 (2003) 9. 